\title{
Complejidad económica y densidad productiva como fuentes de competitividad
}

\author{
VICENTE DONOSO a , VÍCTOR MARTíN ${ }^{\text {b }}$ \\ a Universidad Complutense de Madrid (ICEI), Facultad de Ciencias Económicas y Empresariales, \\ Campus de Somosaguas, 28223 Pozuelo de Alarcón (Madrid), España. E-mail: vdonoso@ccee.ucm.es \\ b Universidad Rey Juan Carlos, (ICEI), Facultad de Ciencias Sociales y Jurídicas, Campus de \\ Vicálvaro, $P^{\circ}$ de los Artilleros, s/n, 28032 Vicálvaro (Madrid), España. E-mail: victor.martin@urjc.es
}

\begin{abstract}
RESUMEN
El análisis de la evolución a largo plazo de las exportaciones españolas muestra un conjunto de rasgos muy positivos. No obstante, ese mismo análisis muestra que subsisten problemas importantes de calidad y de competitividad, sobre todo en comparación con los países más desarrollados. Para mejorar esos resultados es necesario implementar políticas económicas adecuadas. En el trabajo se utilizan los indicadores de complejidad y de densidad productiva como instrumentos para determinar qué productos de la exportación española tienen más posibilidades de expandir su ventaja comparativa revelada o de generar nuevos productos, capaces de competir con ventaja en el comercio internacional. El análisis realizado permite argumentar a favor de políticas sectoriales activas y también formular varias líneas de actuación del sector público, para impulsar el desarrollo de productos con ventaja comparativa.
\end{abstract}

Palabras clave: Exportaciones, complejidad, densidad de productos.

\section{Economic Complexity and Product Density as a Source of Competitiveness}

\begin{abstract}
The analysis of the long-term evolution of Spanish exports shows several positive features. Nevertheless, the analysis shows that there remain significant problems of quality and competitiveness, especially as compared with other developed countries. In order to achieve better results, appropriate economic policies are recommended. In this paper, use is made of an index of economic complexity and product density as instruments to analyze in which products the Spanish economy is more likely to diversify to. The analysis allows to argue in favor of active sectorial policies and also to formulate several lines of action of the public sector, to promote the development of products with comparative advantage.
\end{abstract}

Keywords: Exports, Complexity, Product Density.

Clasificación JEL: F10, F13

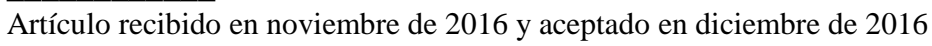

Artículo disponible en versión electrónica en la página www.revista-eea.net, ref. ə-35107 


\section{INTRODUCCIÓN}

El buen comportamiento de las exportaciones es una necesidad fundamental para cualquier economía. Pero además, la creciente globalización económica, con su tendencia a la integración de mercados, convierte la competitividad internacional en una necesidad aún mayor. De manera muy particular, una economía como la de España que tiene una clara inclinación al déficit comercial y a un elevado desempleo, entre otros rasgos, necesita prestarle una atención prioritaria a las principales magnitudes exteriores.

En esta línea de pensamiento, las siguientes páginas se proponen desarrollar algunos enfoques actuales en torno a las ventajas comparativas reveladas del comercio exterior de España y a la forma de extender y ampliar las industrias capaces de competir en el escenario internacional. Para este fin resultan interesantes dos conceptos relativamente recientes: complejidad y densidad de productos. El primero de ellos, permite determinar el grado de complejidad o calidad de los productos exportados, mientras que la densidad de productos caracteriza la facilidad de diversificación hacia nuevos productos, a partir de la estructura productiva existente. De este modo, ambos conceptos ayudan a fundamentar la formulación de políticas económicas conducentes a un incremento de la competitividad exterior.

De acuerdo con este objetivo, el trabajo se compone de los siguientes apartados: en el primero se exponen, desde una perspectiva de largo plazo, algunas de las características de las exportaciones españolas que ayudan a comprender la necesidad de fomentar las actuaciones y las políticas para incrementar la competitividad. En el segundo, se presenta el concepto de complejidad económica y de sus dos componentes, la diversificación y la ubicuidad, además de comentarse la construcción de los índices de complejidad por países y por productos según la metodología propuesta por Hidalgo y Hausman (2009) e Hidalgo (2009). El tercer apartado explica cómo se construye el indicador de proximidad entre productos y se introduce el concepto de densidad, que permite medir la distancia de cada producto respecto de la estructura productiva de un país. Asimismo se combinan los distintos indicadores con el fin de identificar aquellos sectores o productos en los que España tiene una mayor posibilidad de diversificación y que permitirían una mejora en el nivel de complejidad de la economía. En el cuarto apartado se desarrollan algunas implicaciones de política económica. El trabajo se cierra con un resumen de las principales conclusiones.

\section{LAS EXPORTACIONES ESPAÑOLAS}

Como las de muchos otros países, las exportaciones de España oscilan, y a veces fuertemente, en el corto plazo. Piénsese en el período 2008-2011, en el que la tasa de crecimiento de su valor experimenta notables altibajos, que la 
llevan desde el -15,5\% de 2009 hasta el 16,8\% de 2010, pasando en los años inmediatamente anteriores y posteriores por registros también altamente inestables. Este comportamiento cortoplacista puede ocultar los problemas de fondo del comercio y conducir a errores en su diagnóstico y en las políticas adecuadas. Por eso, aquí abordaremos aquellos rasgos de nuestras exportaciones que tienen un carácter más permanente y que revelan con claridad las fortalezas y las debilidades de sus mecanismos competitivos ${ }^{1}$.

\subsection{Rasgos positivos}

Contempladas desde la perspectiva de comienzos de los años sesenta del pasado siglo, las ventas exteriores de España muestran un conjunto de rasgos muy positivos:

a) Continuo aumento de su peso en el PIB; es decir, incremento sostenido de la propensión a exportar. Como se refleja en los siguientes datos: si en 1966 el porcentaje de las exportaciones sobre el PIB era de 4,4\%; en 2015 alcanzó el 23,1\%.

b) También ha aumentado de forma sostenida el peso que dichas ventas representan sobre el total de las exportaciones mundiales. Así, mientras que la cuota de comercio internacional de España era del orden de 0,6 \% al comienzo de los años sesenta del siglo pasado; en 2015, con datos de la OMC, alcanzó el 1,71 \%. Como se ve, casi se han multiplicado por 3, en términos relativos, lo que indica un crecimiento sensiblemente más rápido que la media del comercio mundial. Sin embargo, en este punto hay que introducir una observación relevante: la cuota más alta de comercio la alcanzó España en 2003 con el 2,06\%. A partir de ese año inicia un suave declive (en cualquier caso mucho menos pronunciado que el de otros países importantes de Europa), que indica la necesidad, como se ha comentado en la introducción, de incrementar la competitividad de las ventas de España.

c) El gran aumento cuantitativo ha ido acompañado por una notable transformación cualitativa. Para ilustrar este punto basta con indicar los cambios de peso relativo ocurridos en los grandes sectores económicos. Comparando los datos a lo largo de las más de cinco décadas que median entre 1961 y 2013, podemos destacar lo siguiente: alimentos, bebidas y tabaco han pasado de suponer el $48 \%$ del total exportado a un $15 \%$. Una senda similar aunque menos pronunciada han seguido otros dos grandes sectores materias primas no energéticas más aceites, grasas y ceras vegetales que han disminuido su participación desde un $20 \%$ al 4,2\% del

\footnotetext{
${ }^{1}$ Para lo que se comenta, puede consultarse: Myro et al. (2013), particularmente, las pgs. 311349; Alonso y Donoso (2014); Donoso y Martín (2014); García Tabuenca y Crecente Romero (2014); Ministerior de Economía y Competitividad (2016a).
} 
total. Mencionemos a continuación aquellos sectores que en las décadas analizadas han mantenido su participación prácticamente constante, como son combustibles y lubricantes minerales (con porcentajes en promedio de esos años del $18 \%$ y del 19\%) y artículos manufacturados, clasificados según el material (6\% y $7 \%$ en dicho período). El resto de grandes e importantes sectores económicos han incrementado su peso en las exportaciones, destacando de forma muy visible, maquinaria y equipo de transporte, cuya significación ha pasado del 3,9\% al 34,6\%, lo que lo ha convertido en el grupo con un peso más importante. A continuación, hay que mencionar el sector de productos químicos y conexos, con un relevante salto cuantitativo del 5,2\% al 14,2\%. Y para cerrar, anotaremos que el sector de manufacturas diversas (calzado, muebles, aparatos profesionales) también ha aumentado su peso desde el $4,8 \%$ hasta el $9,7 \%$ del total exportado por España. Un resumen cualitativo de los cambios que se han comentado indicaría que, a lo largo de esas cinco décadas, la economía española ha pasado de un modelo primario exportador al de un país notablemente industrializado, con un peso muy destacado de las industrias de teconología media y media alta.

d) Desde el punto de vista de los mercados de exportación los más de 50 años que transcurren entre 1961 y 2013 también han aportado cambios a la distribución geográfica del comercio español. Sin embargo, estos datos, como se verá por las escuetas cifras que se ofrecerán a continuación, son bastante menos notables que los producidos en la composición por productos. Digamos de forma resumida, que los mercados de la Unión Europea-15, han seguido siendo de forma abrumadora los más importantes, aunque han perdido 4 puntos, hasta situarse en el $56 \%$ del total. Otro gran mercado en el que la presencia española ha sufrido una merma notable es el de los Estados Unidos, que ha cedido seis puntos hasta caer al 3,7\%. Por el contrario, el conjunto de mercados de África (7\%) y Asia (6\%) han incrementado en torno a los dos puntos su participación. Por su parte, América Latina, 50 años después, vuelve a las cuotas de 1961 con un 5\%. Por mercados individuales, Francia (16\%) y Portugal (7,5\%) son los grandes beneficiarios de la reorientación de las ventas de España; en tanto que Alemania (que pierde más de 5 puntos, hasta el 10\%) y Reino Unido (que pierde 10 puntos, hasta el (6,7\%) son los más perjudicados.

e) Mencionaremos también otro rasgo donde el comercio exterior español, visto desde el largo plazo, muestra sus puntos fuertes: el de las empresas exportadoras. El número de empresas que exportan no ha cesado de crecer en las últimas décadas. Si en el quinqueniuo 1987-1991 se censaban 92400 exportadoras, en 2015 ese número había crecido hasta las 147378. Y lo que es más significativo, se había incrementado de forma notable el número de empresas exportadoras regulares. De modo que si, en el citado quinquenio, 
se puede hablar de 17600 empresas (19\% del total de exportadoras); en 2015 este número se estima en $47782(32 \%)^{2}$.

f) No podemos concluir sin aludir al hecho relevante de la presencia del capital extranjero, que ha ido ganando peso a lo largo de las tres últimas décadas, en determinados sectores. Quizá el ejemplo que lo ilustra mejor es el del material de transporte, pero también caucho y plástico, material electrónico u otro equipo eléctrico. Aunque ha habido polémica acerca del efecto neto en la balanza comercial (piénsese por ejemplo en los sectores farmacéuticos, químicos y alimentación y bebidas), poca duda cabe del efecto positivo en las exportaciones brutas y en el cambio estructural de las ventas exteriores de España ${ }^{3}$.

\subsection{Aspectos negativos}

Los aspectos positivos que se acaban de exponer sucintamente coexisten con otros negativos que se mantienen, más allá de mejoras coyunturales, en el largo plazo.

a) Y el primero que debemos mencionar es que persiste la tendencia al déficit comercial, que es uno de los rasgos más permanentes de nuestra economía. Como es sabido, el déficit del comercio de mercancías es el principal responsable de la debilidad de nuestra cuenta corriente y, por tanto, de nuestra necesidad periódica de financiación. En promedio, estamos hablando de un déficit en torno al 5\% del PIB en los últimos 50 años, lo que da una idea de su carácter estructural de largo plazo Esta es una de las principales razones, como se ha mencionado en la introducción de este trabajo, de por qué hay que preocuparse por aumentar la competitividad de nuestras ventas, como el mejor camino para cerrar (junto con los ingresos por turismo) la brecha comercial.

b) A pesar del progreso, hay diferencias cualitativas con países los países más desarrollados. Como se demuestra al analizar la composición de las exportaciones por nivel tecnológico, España es un país de extremos: su ventaja se concentra en productos primarios y manufacturas basadas en recursos naturales, mientras que su desventaja aparece con mucha claridad en las producciones de mayor nivel tecnológico ${ }^{4}$.

c) Otra diferencia cualitativa con el comercio de los países más desarrollados se encuentra en los mecanismos competitivos. España ha progresado, sin

\footnotetext{
${ }^{2}$ En el quinquenio 1987-1991, se entiende por regularidad haber exportado los 5 años; en 2015 el dato se refiere a haber exportado el cuatrienio 2012-2015. Datos de Alonso y Donoso (1994) y Ministerio de Economía y Competitividad (2016b).

${ }^{3}$ Más información en Myro (2013), pp. 330 y ss.

${ }^{4}$ Véase Donoso y Martín (2014), pág. 16.
} 
duda, en los resortes competitivos distintos del precio. Esto es, en la calidad, la incorporación de tecnología o elementos fundamentales del marketing, como es la imagen y marca. Ahora bien, al perder el control del tipo de cambio frente a casi el 55\% de nuestro comercio, conviene insistir en esos mecanismos competitivos de carácter estructural, en lugar de utilizar la disminución de los Índices de Valor Unitario de las exportaciones (IVU) y con ello poner en riesgo el margen empresarial, o la disminución de los Costes Laborales Unitarios (CLU), presionando sobre los salarios nominales ${ }^{5}$. De todos modos, un análisis econométrico de las series 19992011 muestra que el mejor comportamiento de las exportaciones españoles frente a competidores como Francia, Reino Unido, Italia o Alemania, se ha debido no tanto a la evolución a la baja de los indicadores de precios, sino a aquellos factores "residuales" distintos del precio, tales como la calidad del producto o los servicios de distribución y venta ${ }^{6}$.

d) El dato muy positivo en torno a la empresa española, que sin duda muestra una tendencia sostenida en el tiempo a incrementar su presencia exterior y su regularidad, debemos matizarlo con dos observaciones: la primera es que, sobre todo entre las empresas de menos de 50 trabajadores, la probabilidad de exportar sigue siendo notablemente baja en relación a nuestros socios más cercanos, como por ejemplo, Italia. La segunda es el alto nivel de concentración de las exportaciones en un número muy reducido de grandes empresas. Esto es, las 100 mayores exportadoras, de las más de 147000 que venden en el exterior, fueron responsables del $40 \%$ del total exportado en $2015^{7}$. En una perspectiva similar: estudios recientes muestran que el 0,9\% de empresas industriales (del colectivo de empresas mayores de 10 trabajadores) exportan el $51 \%$ del total ${ }^{8}$. Estos datos revelan la necesidad de disminuir la gran dualidad que se aprecia en el conjunto exportador español.

Como se muestra por lo expuesto hasta aquí, más allá de importantes y evidentes mejoras en nuestro comercio, subsisten problemas de fondo que nos deben impulsar a seguir ocupándonos del desarrollo de políticas de competitividad.

\section{COMPLEJIDAD ECONÓMICA}

\subsection{Metodología del índice de complejidad económica}

La metodología propuesta por Hidalgo y Hausman (2009) e Hidalgo (2009)

\footnotetext{
${ }^{5}$ Ambas tendencias pueden corroborarse en Ministerio de Economía y Competitividad (2016c): Análisis de la evolución reciente de los indicadores de competitividad-precio de la economía española(1990-2016).

${ }^{6}$ Véase Correa-López y Domenech (2012), pág.10

${ }^{7}$ Ministerio de Economía y Competitivdad (2016b), pág.39.

${ }^{8}$ Véase Myro (2013), p.326.
} 
permite construir un índice de complejidad de exportaciones, ofreciendo un ranking de países y de productos ordenados en función de su complejidad económica. Partiendo de la idea de que la exportación, y por tanto la producción, de un determinado producto requiere un conjunto de habilidades específicas, la denominada complejidad económica se compone de dos conceptos: diversificación y ubicuidad. Los autores definen la diversificación como el número de productos que un país exporta con ventaja comparativa revelada, mientras que la ubicuidad es el número de países que exportan un determinado producto con ventaja comparativa revelada. De este modo, la diversificación y la ubicuidad constituyen una medida sencilla de la complejidad económica de un país y de un producto respectivamente. Un país será tanto más (menos) complejo, cuanto mayor (menor) sea su diversificación; un producto será tanto más (menos) complejo, cuanto menor (mayor) sea su ubicuidad. Asimismo, la complejidad de un producto representa el conjunto de habilidades específicas requeridas en su producción, mientras que la complejidad de un país hace referencia al conjunto de habilidades disponibles en dicha economía.

En términos prácticos, el primer paso para la obtención de los índices de complejidad económica es el cálculo de la ventaja comparativa revelada (VCR) para cada posible producto y país. Para ello se utiliza el indicador propuesto por Balassa (1965), de manera que la ventaja comparativa revelada del país $c$ en el producto $p$ se obtiene a partir de la siguiente expresión,

$$
V C R_{c p}=\frac{X_{c, p} / X_{c T}}{X_{M, p} / X_{M T}}
$$

en donde $X_{c, p}$ son las exportaciones del país $c$ en el producto $p, X_{c T}$ son las exportaciones totales del país $c, X_{M, p}$ son las exportaciones mundiales en el producto $p, \mathrm{y} X_{M T}$ son las exportaciones mundiales totales. De este modo, la VCR de un país en un determinado producto, se define como el ratio entre el peso de las exportaciones de dicho producto en las exportaciones totales del país y el peso de dicho producto en las exportaciones totales a nivel mundial. Si el valor de VCR es mayor (o igual) que uno para un determinado producto y país, implica que el peso de dicho producto en las exportaciones totales del país es mayor (o igual) que a nivel mundial, en cuyo caso se puede concluir que dicho país tiene un nivel de especialización elevado en dicho producto. Por tanto, cuando el índice es mayor o igual que uno, se considera que el país presenta ventaja comparativa revelada en la exportación de un determinado producto. Intuitivamente, si un país es capaz de exportar un producto con ventaja comparativa revelada, se debe a que posee las capacidades necesarias y específicas, tales como los conocimientos adquiridos por los trabajadores, las instituciones necesarias, maquinaria específica, y los insumos públicos entre otras. De este modo, un país con un alto grado de diversificación, presenta un conjunto amplio de capacidades específicas, mientras que un producto con un 
nivel bajo de ubicuidad, requiere de un conjunto más exclusivo de capacidades. En consecuencia, el concepto de complejidad, está asociado al conjunto de capacidades específicas que posee un determinado país (complejidad económica por país), y al conjunto de capacidades requeridas en la producción de un determinado producto (complejidad económica por producto).

Una vez obtenidos los indicadores de ventaja comparativa revelada para cada país y producto, los conceptos de diversificación y ubicuidad son calculados, en términos formales, de la siguiente manera,

$$
\begin{gathered}
k_{c, 0}=\sum_{p=1}^{N_{p}} M_{c p} \quad \text { (Diversificación) } \\
k_{p, 0}=\sum_{c=1}^{N_{c}} M_{c p} \quad \text { (Ubicuidad) }
\end{gathered}
$$

en donde de nuevo $c$ denota país, $p$ denota producto y $M_{c p}$ toma valor uno si la ventaja comparativa revelada del país $c$ en el producto $p$ es mayor o igual a la unidad, y cero en caso contrario. Por su parte, $N_{p}$ y $N_{c}$ son el número total de productos exportados por el país $c$ y el número total de países que exportan el producto $p$ respectivamente. La información obtenida sobre diversificación por países y ubicuidad por producto se relaciona entre sí para obtener sendos indicadores de complejidad por país y por producto calculando de forma iterativa las siguientes expresiones,

$$
\begin{aligned}
& k_{c, N}=\frac{1}{k_{c, 0}} \sum_{p=1}^{N_{p}} M_{c p} k_{p, N-1} \\
& k_{p, N}=\frac{1}{k_{p, 0}} \sum_{c=1}^{N_{c}} M_{c p} k_{c, N-1}
\end{aligned}
$$

Este proceso iterativo es lo que los autores denominan "Method of reflections". Cuando el valor obtenido de $k_{c, N} \mathrm{y} k_{p, N}$ en una determinada iteración no presenta grandes cambios respecto de la iteración previa, el proceso se detiene dando lugar a dos indicadores de complejidad económica refinados. Es decir, a medida que el número de iteraciones se va incrementando, los indicadores resultantes tienden a su valor medio, de manera que cuando en una determinada iteración no se observan cambios significativos respecto de la iteración previa, podemos concluir que el proceso ha extraído toda la información posible, por lo que iteraciones adicionales no permiten obtener ninguna información adicional.

\subsection{Complejidad Económica: el caso de España}

Los datos empleados para el cálculo del índice de complejidad económica y la obtención del ranking de países y productos según su grado de complejidad 
para el periodo 1995-2014 proceden de la base COMTRADE de Naciones Unidas. Todos los datos están expresados en dólares de los Estados Unidos, siguiendo la clasificación CUCI Revisión 3 a 4 dígitos. Siguiendo las recomendaciones de Hidalgo y Hausman (2009) e Hidalgo (2009), y con el fin de depurar la base de datos y obtener indicadores de complejidad lo más fiables posibles, se han seleccionado aquellos países para los que se dispone de datos de comercio desagregados por producto, con una población superior a 1.200 .000 y que exportaron, en promedio, al menos por valor de 1.000 millones de euros anuales a lo largo del periodo 1995-2014. Asimismo y con el objetivo de poder hacer comparaciones a lo largo del periodo considerado del ranking de productos y países, se ha mantenido un mundo constante. Es decir, se han incluido solo aquellos países de los que se dispone de datos de exportaciones para todos y cada uno de los años dentro del periodo de análisis. Una vez depurada la base de datos, contamos con un total de 100 países y 1.009 productos.

Siguiendo la metodología expuesta previamente para el cálculo de los indicadores de complejidad económica, el primer paso realizado ha sido la obtención de la VCR para cada país y producto a lo largo del periodo 1995-2014. De este modo es posible conocer la diversificación, o número de productos exportados con ventaja comparativa, para cada uno de los países de la muestra y por tanto tener una primera aproximación sobre la complejidad económica por países.

La Tabla 1 muestra la diversificación de España y de los distintos países de la UE-15 (con la excepción de Luxemburgo) para diversos años del periodo analizado, junto con el valor promedio para la UE-15 y el mundo. La información presentada muestra al menos dos cuestiones de interés. En primer lugar, el número de productos que España exporta con ventaja comparativa revelada se ha incrementado notablemente a lo largo del periodo considerado. Pues en efecto, si bien en el año 1995 España exportó un total de 317 productos con ventaja comparativa revelada, en el año 2014 el número de productos fue de 383 , lo que supone un incremento de algo más un 20 por ciento. En el conjunto de los países de nuestro entorno (UE-15) y a nivel mundial, el incremento ha sido mucho más leve, ya que en promedio, el número de productos exportados por dicho conjunto de países se ha mantenido en el entorno de 310 y de 176 productos respectivamente, con un incremento del 6,1\% y una caída del 3,4\% respectivamente.

En segundo lugar, España presenta un nivel de diversificación elevado, tanto si nos comparamos con el conjunto de países de la UE-15 como con el conjunto de países a nivel mundial. Atendiendo a los datos correspondientes al 2014, se observa que España es uno de los países con mayor grado de diversificación, ocupando el cuarto puesto por detrás de Italia, Alemania y Francia. Por tanto, España no solo presenta un número relativamente elevado de productos en los 
que tiene un grado de especialización alto, sino que ha realizado un gran esfuerzo durante las últimas dos décadas en lo que se refiere a la especialización en nuevos productos.

Tabla 1

Diversificación en los países de la UE-15, 1995-2014

\begin{tabular}{|l|c|c|c|c|c|c|}
\hline \multicolumn{1}{|c|}{ País } & $\mathbf{1 9 9 5}$ & $\mathbf{2 0 0 0}$ & $\mathbf{2 0 0 5}$ & $\mathbf{2 0 1 0}$ & $\mathbf{2 0 1 4}$ & $\begin{array}{c}\text { Variación } \\
\mathbf{1 9 9 5 - 2 0 1 4} \\
\text { (\%) }\end{array}$ \\
\hline Alemania & 447 & 441 & 431 & 438 & 428 & -4.3 \\
\hline Austria & 343 & 336 & 335 & 356 & 368 & 7.3 \\
\hline Bélgica & - & 365 & 320 & 342 & 336 & - \\
\hline Dinamarca & 268 & 305 & 276 & 302 & 302 & 12.7 \\
\hline España & $\mathbf{3 1 7}$ & $\mathbf{3 5 5}$ & $\mathbf{3 8 5}$ & $\mathbf{3 9 3}$ & $\mathbf{3 8 3}$ & $\mathbf{2 0 . 8}$ \\
\hline Finlandia & 200 & 192 & 219 & 232 & 209 & 4.5 \\
\hline Francia & 412 & 425 & 417 & 404 & 397 & -3.6 \\
\hline Grecia & 217 & 247 & 258 & 238 & 192 & -11.5 \\
\hline Irlanda & 171 & 127 & 119 & 120 & 125 & -26.9 \\
\hline Italia & 372 & 428 & 425 & 462 & 449 & 20.7 \\
\hline Países Bajos & 334 & 277 & 287 & 298 & 354 & 6.0 \\
\hline Portugal & 214 & 265 & 272 & 337 & 328 & 53.3 \\
\hline Reino Unido & 351 & 285 & 288 & 276 & 289 & -17.7 \\
\hline Suecia & 247 & 267 & 265 & 283 & 289 & 17.0 \\
\hline UE-15 & $\mathbf{2 9 9}$ & $\mathbf{3 0 8}$ & $\mathbf{3 0 7}$ & $\mathbf{3 2 0}$ & $\mathbf{3 1 8}$ & $\mathbf{6 . 1}$ \\
\hline Mundo & $\mathbf{1 7 9}$ & $\mathbf{1 7 5}$ & $\mathbf{1 7 7}$ & $\mathbf{1 7 6}$ & $\mathbf{1 7 3}$ & $\mathbf{- 3 . 4}$ \\
\hline
\end{tabular}

Notas: Luxemburgo no se incluye en el análisis. Los datos correspondientes a UE-15 y al Mundo consisten en valores medios.

Fuente: Elaboración propia a partir de la base de datos de UN COMTRADE.

Una vez calculados los indicadores de VCR, se ha procedido a calcular el índice de complejidad económica por países y por productos siguiendo el método iterativo previamente expuesto. En el análisis, hemos utilizado $k_{c, 16}$ como medida de la complejidad económica por países y $k_{p, 17}$ como medida de complejidad económica por productos (ver Hidalgo y Hausmann (2009) y Hidalgo (2009) para mayor detalle). Los resultados del índice de complejidad económica por países para el año 2014 se presentan en la Tabla 2. Dicha tabla presenta el conjunto de países de la muestra ordenados según el ranking de complejidad de mayor a menor, así como su variación respecto del año 2000.

Como puede apreciarse, los diez primeros puestos los ocupan Japón, Suiza, Alemania, Corea del Sur, Singapur, Suecia, Austria, Finlandia, República Checa y Reino Unido. Además, con la excepción de Corea del Sur y Singapur que han ganado 11 y 7 puestos respectivamente, estos países han mantenido su posición en el ranking desde el año 2000 relativamente estable. Entre los principales productos exportados por estos países destacan los vehículos automotores (7812) que representan el 12,8\% de las exportaciones totales en Japón, el 10,7\% en 
Alemania, el 10,2\% en la República Checa, el 8,2\% en el Reino Unido y el 7,8\% en Corea del Sur. Asimismo destacan los Medicamentos (5429), cuyo peso sobre el total de exportaciones en la República Checa alcanza un 10,1\% y un $4 \%$ en el Reino Unido, así como otras partes, piezas y accesorios de los vehículos automotores (7843), con un peso del 7,6\% en República Checa y cercano al 5\% en Alemania, Japón y Corea del Sur.

Tabla 2

Índice de complejidad económica de las exportaciones por países, 2014

\begin{tabular}{|c|c|c|c|c|c|c|c|}
\hline Ranking & Variación & País & Código & Ranking & Variación & País & Código \\
\hline 1 & 0 & Japón & JPN & 51 & 13 & El Salvador & SLV \\
\hline 2 & 1 & Suiza & $\mathrm{CHE}$ & 52 & 4 & Colombia & $\mathrm{COL}$ \\
\hline 3 & -1 & Alemania & DEU & 53 & -3 & Grecia & GRC \\
\hline 4 & 11 & Corea del Sur & KOR & 54 & 6 & Rep. de Macedonia & MKD \\
\hline 5 & 7 & Singapur & SGP & 55 & -3 & Uruguay & URY \\
\hline 6 & -2 & Suecia & SWE & 56 & 28 & Vietnam & VNM \\
\hline 7 & 0 & Austria & AUT & 57 & 4 & Indonesia & IDN \\
\hline 8 & -3 & Finlandia & $\mathrm{FIN}$ & 58 & -11 & Argentina & ARG \\
\hline 9 & 1 & Rep. Checa & CZE & 59 & 13 & Honduras & HND \\
\hline 10 & -4 & Reino Unido & GBR & 60 & -9 & Kuwait & KWT \\
\hline 11 & 3 & Eslovenia & SVN & 61 & 13 & Egipto & EGY \\
\hline 12 & -4 & Estados Unidos & USA & 62 & -5 & Moldavia & MDA \\
\hline 13 & 12 & Hungría & HUN & 63 & 6 & Guatemala & GTM \\
\hline 14 & 2 & Eslovaquia & SVK & 64 & -6 & Chile & $\mathrm{CHL}$ \\
\hline 15 & -4 & Italia & ITA & 65 & 10 & Panamá & PAN \\
\hline 16 & -7 & Francia & FRA & 66 & 1 & Senegal & SEN \\
\hline 17 & 2 & Israel & ISR & 67 & -18 & Australia & AUS \\
\hline 18 & -5 & Irlanda & $\mathrm{IRL}$ & 68 & 12 & Sri Lanka & LKA \\
\hline 19 & 1 & Dinamarca & DNK & 69 & 23 & Camerún & CMR \\
\hline 20 & -3 & Bélgica & BEL & 70 & 13 & Jamaica & JAM \\
\hline 21 & 5 & México & MEX & 71 & 7 & Zambia & ZMB \\
\hline 22 & 10 & China & $\mathrm{CHN}$ & 72 & 24 & Malaui & MWI \\
\hline 23 & 4 & Polonia & $\mathrm{POL}$ & 73 & 4 & Mauricio & MUS \\
\hline 24 & -2 & Países Bajos & NLD & 74 & -8 & $\begin{array}{l}\text { Emir. Árabes } \\
\text { Unidos }\end{array}$ & ARE \\
\hline 25 & 15 & Estonia & EST & 75 & -12 & Omán & OMN \\
\hline 26 & 7 & Hong Kong & HKG & 76 & 12 & Marruecos & MAR \\
\hline 27 & -3 & Noruega & NOR & 77 & -47 & Georgia & GEO \\
\hline 28 & 7 & Malasia & MYS & 78 & 19 & Uganda & UGA \\
\hline 29 & 5 & Croacia & HRV & 79 & -11 & Albania & ALB \\
\hline 30 & 15 & Tailandia & THA & 80 & -25 & Kazajistán & $\mathrm{KAZ}$ \\
\hline 31 & 7 & Rumanía & ROM & 81 & -5 & Paraguay & PRY \\
\hline 32 & 11 & Lituania & LTU & 82 & 3 & Pakistán & PAK \\
\hline 33 & -5 & Bielorrusia & BLR & 83 & -4 & Perú & PER \\
\hline 34 & -13 & Canadá & CAN & 84 & -14 & Zimbabue & ZWE \\
\hline 35 & -12 & España & ESP & 85 & 4 & Costa de Marfil & CIV \\
\hline 36 & 1 & Letonia & LVA & 86 & 7 & Madagascar & MDG \\
\hline
\end{tabular}


Tabla 2 (Continuación)

Índice de complejidad económica de las exportaciones por países, 2014

\begin{tabular}{|c|c|l|c|c|c|l|c|}
\hline Ranking & Variación & \multicolumn{1}{|c|}{ País } & Código & Ranking & Variación & País & Código \\
\hline 37 & -1 & Portugal & PRT & 87 & 0 & Camboya & KHM \\
\hline 38 & 15 & Turquía & TUR & 88 & -2 & Mozambique & MOZ \\
\hline 39 & -8 & Ucrania & UKR & 89 & -7 & Nicaragua & NIC \\
\hline 40 & 4 & Bulgaria & BGR & 90 & 4 & Tanzania & TZA \\
\hline 41 & 7 & Líbano & LBN & 91 & -29 & Azerbaiyán & AZE \\
\hline 42 & 23 & Filipinas & PHL & 92 & -2 & Ecuador & ECU \\
\hline 43 & 3 & Jordania & JOR & 93 & -20 & Bolivia & BOL \\
\hline 44 & 15 & Túnez & TUN & 94 & -23 & Argelia & DZA \\
\hline 45 & -6 & Arabia Saudita & SAU & 95 & -14 & Mongolia & MNG \\
\hline 46 & 8 & India & IND & 96 & 3 & Etiopía & ETH \\
\hline 47 & -6 & Nueva Zelanda & NZL & 97 & 1 & Catar & QAT \\
\hline 48 & -19 & Brasil & BRA & 98 & -7 & Mauritania & MRT \\
\hline 49 & -31 & Rusia & RUS & 99 & 1 & Nigeria & NGA \\
\hline 50 & -8 & Sudáfrica & ZAF & 100 & -5 & Guinea & GIN \\
\hline
\end{tabular}

Notas: Variación = variación en el puesto en 2014 respecto del año 2000. Un valor negativo implica una caída en el ranking.

Fuente: Elaboración propia a partir de la base de datos de UN COMTRADE.

Por su parte, España ocupa el puesto 35, con una pérdida de12 puestos desde el año 2000. A pesar de que España se sitúa muy por encima de la mediana en el ranking, en comparación con los países de nuestro entorno más cercano (UE15), se observa que, con la excepción de Portugal y Grecia, España ocupa un puesto inferior, y muy alejado de algunos de estos países como es el caso de Alemania (puesto 3), Suecia (puesto 6), Austria, (puesto 7), Finlandia (puesto 8) y Reino Unido (puesto 10). Este hecho contrasta sin embargo, con el alto grado de diversificación de España y el considerable aumento en el número de productos exportados con ventaja comparativa revelada durante las últimas dos décadas. La explicación a estos resultados tan dispares, podría obtenerse analizando la calidad o complejidad económica de los productos exportados por España con ventaja comparativa revelada.

A modo ilustrativo, en la Figura 1 se muestra la ubicuidad media de los productos exportados con ventaja comparativa revelada en el eje de ordenadas, frente a la diversificación en el eje de abscisas para el conjunto total de países de la muestra. Las líneas de división vertical y horizontal muestran la diversificación y la ubicación media en promedio del conjunto de países considerados, lo que nos permite dividir la figura en cuatro cuadrantes distintos. España se sitúa en el cuadrante formado por el conjunto de países con un grado de diversificación por encima de la media, y cuya ubicuidad media se encuentra por debajo del promedio a nivel mundial. Es decir, países que exportan un número de productos con ventaja comparativa revelada por encima de la media y en donde la calidad media de los productos exportados se sitúa por encima de la media. Ahora bien, 
en comparación con los países de la UE-15, se observa que en su mayoría, la ubicuidad media de estos países es inferior a la de España, lo que sugiere en principio una mayor calidad o complejidad de los productos exportados por dichos países.

Figura 1

Diversificación y ubicuidad media, 2014

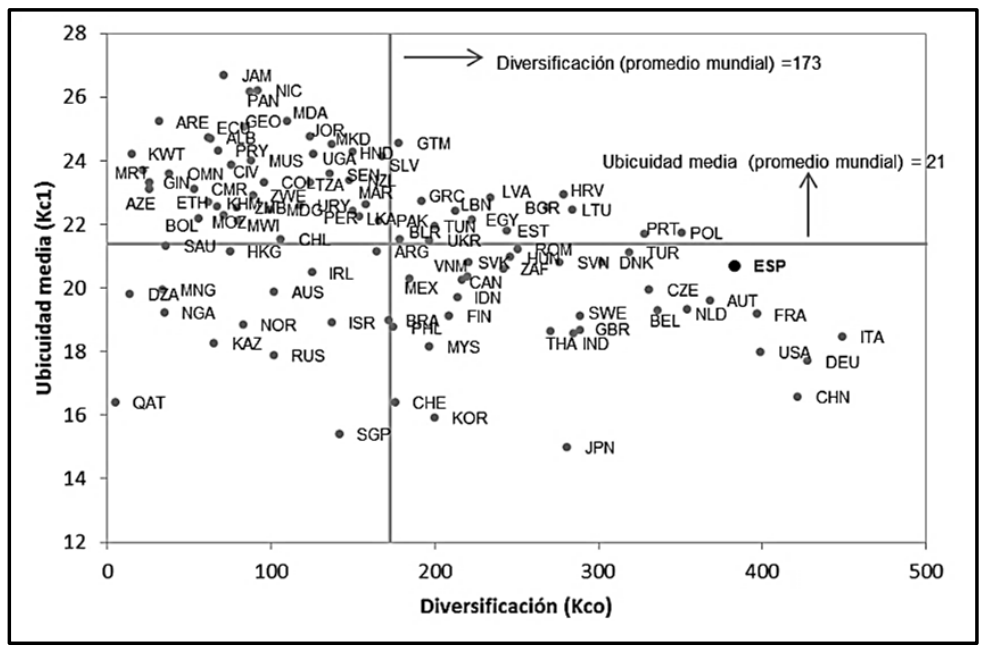

Fuente: Elaboración propia a partir de la base de datos de UN COMTRADE.

Con el fin de profundizar más en la calidad de los productos exportados, se ha procedido a clasificar la cesta exportadora de los distintos países en función del grado de complejidad económica de los productos. En base a la información proporcionada por el índice de complejidad por productos se han calculado los distintos quintiles de la distribución, lo que permite clasificar los productos en cinco grupos distintos: complejidad alta, complejidad media-alta, complejidad media, complejidad media-baja y complejidad baja. La Tabla 3 muestra la estructura de la cesta de exportaciones de España $^{9}$ y del resto de países de la UE-15, en función del grado de complejidad económica.

Los datos de la Tabla 3 muestran diversas cuestiones de interés. En primer lugar, la categoría de exportaciones de complejidad media-alta (Q4) es la de mayor peso pues concentra el 28,6\% de las exportaciones españolas, seguida de la categoría de exportaciones de complejidad media-baja (Q2), cuyo peso sobre el total de exportaciones fue de un 22,4\%. A esta le sigue la categoría de complejidad alta (Q5) con un peso del 16,7\%. Dentro de esta categoría, el $22,5 \%$ de productos son exportados con ventaja comparativa revelada, si bien

\footnotetext{
${ }^{9}$ Un análisis similar para el año 2000 puede encontrarse en Minondo (2007).
} 
los productos con un mayor peso relativo sobre el total de las exportaciones son otras partes, piezas y accesorios de vehículos automotores (3,4\%; cod. 7843), los motores de combustión interna, de émbolo para la propulsión de vehículos (1\%; cod. 7132) y glucósidos, glándulas u otros órganos y sus extractos, antisueros, vacunas y productos análogos (0,9\%; cod. 5416) . Por su parte, dentro de la categoría de exportaciones de complejidad media-alta, el $41 \%$ de los productos que la componen son exportados por España con ventaja comparativa revelada. Los productos que presentan un mayor peso relativo sobre el total de las exportaciones son los vehículos automotores para el transporte de personas (10\%; cod. 7812), los vehículos automotores para el transporte de mercancías (1,9\%; cod. 7821) y partes y piezas de aeronaves (1\%; cod. 7929). La categoría de complejidad media representa el $15,7 \%$ de las exportaciones totales, dentro de la cual el 39,2\% de los productos que la componen son exportados con ventaja comparativa revelada. Las categorías de complejidad media-baja (Q2) y complejidad baja (Q1) suponen un 22,4\% y 16,5\% de las exportaciones totales respectivamente. Dentro de dichas categorías, el 51,5\% y el 39,6\% del total de productos que las componen, son productos exportados con ventaja comparativa revelada.

\section{Tabla 3}

Composición de las exportaciones de bienes por complejidad, 2014

\begin{tabular}{|l|c|c|c|c|c|}
\hline \multirow{2}{*}{ País } & \multicolumn{5}{|c|}{ Nivel de complejidad } \\
\cline { 2 - 6 } & $\begin{array}{c}\text { baja } \\
\text { (Q1) }\end{array}$ & $\begin{array}{c}\text { media-baja } \\
\text { (Q2) }\end{array}$ & $\begin{array}{c}\text { media } \\
\text { (Q3) }\end{array}$ & $\begin{array}{c}\text { media-alta } \\
\text { (Q4) }\end{array}$ & $\begin{array}{c}\text { alta } \\
\text { (Q5) }\end{array}$ \\
\hline Alemania & 3.8 & 14.8 & 15.6 & 34.1 & 31.6 \\
\hline Austria & 4.5 & 17.4 & 18.1 & 27.1 & 33.0 \\
\hline Bélgica & 12.3 & 22.1 & 20.9 & 22.6 & 22.1 \\
\hline Dinamarca & 11.4 & 23.8 & 18.6 & 26.1 & 20.1 \\
\hline España & $\mathbf{1 6 . 5}$ & $\mathbf{2 2 . 4}$ & $\mathbf{1 5 . 7}$ & $\mathbf{2 8 . 6}$ & $\mathbf{1 6 . 7}$ \\
\hline Finlandia & 2.6 & 30.2 & 16.8 & 27.1 & 23.3 \\
\hline Francia & 7.1 & 18.8 & 20.8 & 29.6 & 23.7 \\
\hline Grecia & 15.9 & 52.9 & 15.1 & 12.3 & 3.8 \\
\hline Irlanda & 2.9 & 10.9 & 23.6 & 13.7 & 48.9 \\
\hline Italia & 9.1 & 21.3 & 18.6 & 23.6 & 27.3 \\
\hline Países Bajos & 13.8 & 27.4 & 18.9 & 18.3 & 21.5 \\
\hline Portugal & 16.5 & 28.0 & 16.9 & 24.5 & 14.0 \\
\hline Reino Unido & 17.9 & 15.9 & 16.8 & 26.6 & 22.7 \\
\hline Suecia & 6.9 & 24.6 & 18.3 & 23.4 & 26.7 \\
\hline UE-15 & $\mathbf{9 . 2}$ & $\mathbf{1 9 . 7}$ & $\mathbf{1 7 . 9}$ & $\mathbf{2 7 . 1}$ & $\mathbf{2 6 . 1}$ \\
\hline Mundo & $\mathbf{2 1 . 0}$ & $\mathbf{2 0 . 3}$ & $\mathbf{1 5 . 2}$ & $\mathbf{2 1 . 9}$ & $\mathbf{2 1 . 6}$ \\
\hline
\end{tabular}

Notas: Luxemburgo no se incluye en el análisis.

Fuente: Elaboración propia a partir de la base de datos de UN COMTRADE.

En segundo lugar, si comparamos la estructura exportadora por nivel de complejidad de España con los principales países de nuestro entorno, se 
corrobora que España presenta un nivel de complejidad por debajo de la media. Pues en efecto, si bien en el caso de las exportaciones de complejidad mediaalta, el peso que representan en España (28,6\%) se sitúa levemente por encima de la media de los países de la UE-15 (27,1\%), en el caso de las exportaciones de complejidad alta las diferencias son significativamente notables. Con la excepción de Grecia y Portugal, el peso relativo de las exportaciones de complejidad alta en España (16,7\%) es inferior al del resto de países, en donde en diversos casos, el peso de dichas exportaciones alcanza y supera prácticamente la tercera parte de las exportaciones totales, como es el caso de Irlanda (48,9\%), Austria (33\%) y Alemania (31,6\%). Además, en el caso de las exportaciones de complejidad baja (16,5\%), el peso que representan en España es muy superior al del promedio de la UE-15(9,2\%).

La Figura 2 presenta la evolución de la estructura exportadora de España por grado de complejidad a lo largo del periodo analizado. Como puede apreciarse, el peso de las exportaciones de complejidad alta se ha reducido considerablemente desde el año 1995, con una pérdida de 19 puntos porcentuales, mientras que el peso de las exportaciones de complejidad baja y complejidad media-baja se ha incrementado en 2,2 y 6,8 puntos porcentuales respectivamente. Como buena noticia, destaca el importante incremento en el peso de las exportaciones de complejidad media-alta, pues han pasado desde el $18,9 \%$ en 1995 hasta el $28,6 \%$ en 2014, lo que implica un incremento de casi 10 puntos porcentuales.

Figura 2

Estructura de las exportaciones de España por complejidad, 1995, 2000 y 2014

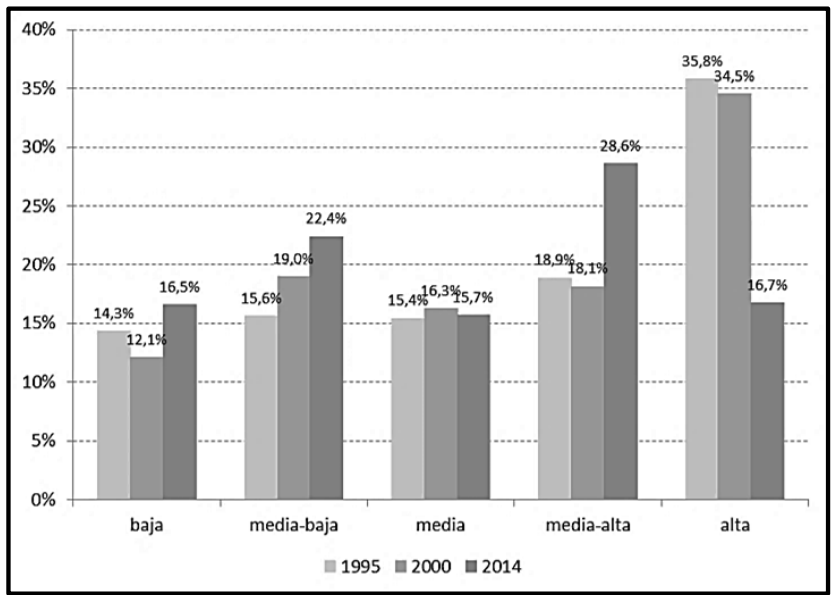

Fuente: Elaboración propia a partir de la base de datos de UN COMTRADE.

Los distintos resultados obtenidos a partir del análisis realizado en el presente apartado permiten obtener al menos dos conclusiones importantes. En 
primer lugar, España ha realizado un gran esfuerzo exportador durante las últimas dos décadas, periodo durante el cual el número de productos que España exporta con ventaja comparativa revelada se ha incrementado de forma notable. Por tanto, España goza de un alto grado de diversificación dentro de su cesta exportadora. En segundo lugar, y a pesar del gran esfuerzo exportador, el grado de complejidad económica de las exportaciones españolas se mantiene inferior al de los principales países de nuestro entorno. Así, España ocupa un puesto muy por debajo de los principales países de la Unión Europea en el índice de complejidad, puesto este que se ha deteriorado durante las últimas décadas. Además, el peso que representan las exportaciones de complejidad alta sobre el total de exportaciones, se ha reducido de forma considerable alcanzando valores muy inferiores a los observados en los países de nuestro entorno. Ahora bien, estos resultados han de interpretarse con cierta cautela, pues en ningún caso implican una pérdida de calidad o complejidad de las exportaciones españolas en términos absolutos, aunque si en términos relativos. Los resultados ponen de manifiesto, que los esfuerzos realizados por España en materia de diversificación y mejora de la calidad de la cesta exportadora no han sido suficientes y que con el fin de mejorar el grado de complejidad de la economía española, se deberían realizar esfuerzos adicionales en la especialización en productos con un mayor grado de complejidad.

\section{DENSIDAD DE PRODUCTOS Y DIVERSIFICACIÓN ECONÓMICA}

\subsection{Proximidad entre productos y densidad: conceptos y metodología}

Desde un punto de vista teórico, el concepto de proximidad o cercanía entre productos o industrias, hace referencia al grado de similitud en el conjunto de conocimientos, habilidades y capacidades requeridos para la producción de determinados productos o en determinadas industrias. Es decir, dos productos serán más próximos entre sí, cuanto mayor sea la similitud en el conjunto de conocimientos y habilidades requeridos para su producción, o la similitud en la tecnología de producción requerida.

Con el fin de analizar la proximidad entre productos se ha empleado el índice de proximidad propuesto por Hidalgo et al. $(2007)^{10}$. El índice, parte de la idea de que si dos productos diferentes están relacionados entre sí, como consecuencia de que su producción requiere de factores de producción similares, conocimientos, infraestructuras o instituciones parecidas, tenderán a ser producidos dentro de la misma región o país. Sin embargo, si los productos no están relacionados entre

${ }^{10}$ Otros métodos para analizar la proximidad o relación entre productos pueden encontrarse en los trabajos de Farjoun (1994), Teece et al. (1994), Fan y Lang (2000), Nefke y Henning (2008), Bryce y Winter (2009) y Neffke et al. (2011). 
sí, será menos probable que sean producidos conjuntamente dentro de una misma región. A efectos prácticos, el índice de proximidad entre dos bienes se calcula como el valor mínimo de tener ventaja comparativa revelada en cada uno de ellos, es decir,

$$
\varphi_{i, j, t}=\min \left\{P\left(V C R x_{i, t} \mid V C R x_{j, t}\right), P\left(V C R x_{j, t} \mid V C R x_{i, t}\right)\right\}
$$

en donde $P\left(V C R x_{i, t} \mid V C R x_{j, t}\right)$ es la probabilidad condicionada de que un país tenga ventaja comparativa revelada en el bien $i$ dado que el país tiene ventaja comparativa revelada en el bien $j$. Dicha probabilidad condicionada se calcula como el cociente entre la probabilidad conjunta de tener ventaja comparativa revelada en el bien $i$ y en el bien $j$, y la probabilidad de tener ventaja comparativa revelada en el producto $j$. La probabilidad conjunta de tener ventaja comparativa revelada en el bien $i$ y en el bien $j$ se ha calculado dividiendo el número de países de la muestra que tienen ventaja comparativa revelada en ambos bienes y el número total de países de la muestra. Por su parte, la probabilidad de tener ventaja comparativa revelada en un bien $i$ se ha calculado como el cociente entre el número de países que tienen ventaja comparativa en dicho bien, y el total de países en la muestra. De forma similar se ha calculado $P\left(V C R x_{j, t} \mid V C R x_{i, t}\right)$, es decir, la probabilidad condicionada de que un país tenga ventaja comparativa revelada en el bien $j$ dado que el país tiene ventaja comparativa revelada en el bien $i$.

A partir de los valores obtenidos de proximidad tecnológica entre productos, es posible calcular un indicador de densidad que capte el grado de relación entre cada uno de los productos exportados por un país o región y la estructura productiva existente en dicho país o región. Siguiendo el trabajo de Hausman y Klinger (2007), es posible calcular dicho índice de densidad como la suma del indicador de proximidad de un determinado producto $i$ respecto de todos aquellos productos exportados por el país o región con ventaja comparativa revelada, dividido entre la suma del indicador de proximidad del producto $i$ respecto de todos los posibles productos. Algebraicamente, la densidad de un país $c$, en un producto $i$ se calcula a partir de la siguiente expresión,

$$
\operatorname{densidad}_{i, c, t}=\frac{\sum_{j} \varphi_{i, j, t} x_{c, j, t}}{\sum_{j} \varphi_{i, j, t}}
$$

en donde $\varphi_{i, j, t}$ es el índice de proximidad entre el producto $i$ y el producto $j$ definido en la ecuación (6), y $x_{c, j, t}$ toma valor uno si el país o región $c$ tiene ventaja comparativa revelada en el producto $j$ y valor cero en caso contrario. El indicador de densidad toma valores entre cero y uno. Si un determinado país exporta todos los productos próximos al producto $i$ con ventaja comparativa revelada, la densidad tomará valor uno. Sin embargo, si el país en cuestión no 
tiene ventaja comparativa revelada en ninguno de los productos próximos al producto $i$, la densidad tomará valor cero. De este modo, si un país o región presenta ventaja comparativa revelada en la mayoría de productos relacionados con el producto $i$, la densidad será elevada, y la probabilidad de que el país desarrolle ventaja comparativa revelada en dicho producto en el futuro será alta. Además, y tal como han demostrado algunos estudios empíricos (Hausman y Klinger, 2007; Boschma et al., 2012), si un país o región presenta ventaja comparativa revelada en la mayoría de productos relacionados con un determinado producto que ya está siendo exportado con ventaja comparativa revelada, la probabilidad de mantener dicha ventaja comparativa será mayor.

\subsection{Densidad de productos y posibilidades de diversificación en España}

A partir de las estadísticas de comercio exterior procedentes de la base COMTRADE de Naciones Unidas y partiendo de nuevo de la clasificación CUCI Revisión 3 a 4 dígitos, se ha procedido a calcular en primer lugar el índice de proximidad para cada uno de los posibles pares de bienes de la muestra en el año 2014. A modo ilustrativo, la Figura 3 muestra los 50 productos con un mayor grado de proximidad, mediante un gráfico de redes, a los vehículos automotores para el transporte de personas (cod. 7812), producto este que ha tenido tradicionalmente un peso relativo elevado en el conjunto de exportaciones totales de España, y que en el año 2014 representó el 10\%. En la figura, los nodos más cercanos a los vehículos presentan un mayor grado de proximidad. Asimismo, los nodos de mayor tamaño implican la existencia de ventaja comparativa revelada en el caso de España en el año 2014, mientras que las diferencias en forma y color clasifican los productos en función de diferencias en su código a un dígito o número de sección.

Como puede apreciarse, los productos con un mayor grado de proximidad a los vehículos para el transporte de personas son otras partes, piezas y accesorios de vehículos (prox. $=0,64$; cod. 7843), árboles de transmisión y manivelas (prox. $=0,58$; cod. 7481), partes y piezas de los motores de combustión interna de émbolo (prox.=0,58; cod. 7139), bombas de aire o de vacío, compresores y campanas de ventilación (prox. $=0,58$; cod. 7431 ) y los vehículos automotores para el transporte de mercancías (prox. $=0,54$; cod. 7821). Cabe destacar a su vez que la gran mayoría de productos, aunque no todos, comparten el mismo valor de código a un dígito que los vehículos para el transporte de mercancías. De forma más específica, el $70 \%$ de los productos pertenecen a la sección 7 (maquinaria y equipo de transporte), mientras que un $20 \%$ pertenecen a la sección 6 (artículos manufacturados). A su vez, dentro de la sección 7, el 60\% pertenecen al grupo, a dos dígitos, número 74 (maquinaria y equipo industrial en general y partes y piezas de máquinas), mientras que dentro de los productos de la sección 6, nos encontramos principalmente con productos del grupo 
número 62 (manufacturas de caucho) y 69 (manufacturas de metales). Por último, cabe destacar que España mantiene ventaja comparativa revelada en el $48 \%$ de los productos más próximos a los vehículos para el transporte de personas representados en la figura.

Figura 3

Los 50 productos más próximos a los vehículos (cod. 7812), 2012

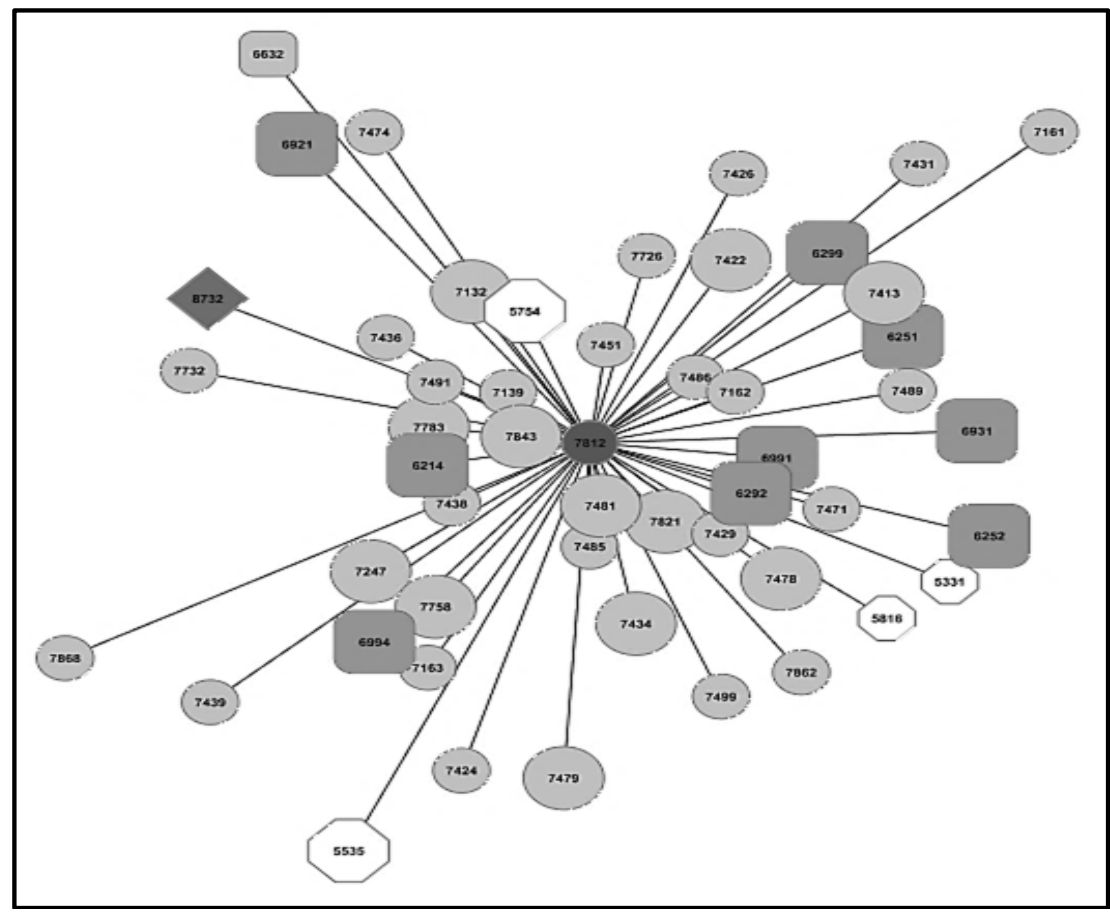

Fuente: Elaboración propia a partir de la base de datos UN COMTRADE, mediante el programa Cytoscape.

Mediante el uso de los datos previamente obtenidos de proximidad tecnológica entre productos, y las ventajas comparativas reveladas obtenidas a partir de la base de datos COMTRADE, se ha procedido a calcular para el año 2014, la densidad de cada producto en el caso de España. De este modo, para cada posible producto disponemos de su grado de especialización, medido por el índice de VCR, su posibilidad de diversificación, medida por el grado de densidad, y su nivel de complejidad, lo que permite obtener la relación de productos en los que España puede diversificarse con una mayor probabilidad según las diferentes categorías de complejidad.

La Figura 4 muestra, para productos de complejidad alta, el grado de especialización o índice de VCR en el eje de ordenadas, frente a la la densidad o distancia de los productos respecto de la estructura productiva existente, 
representada en el eje de abcisas, para el conjunto de productos exportados por España en el año 2014. Unicamente se representan aquellos productos con una ventaja comparativa revelada inferior a uno, es decir, aquellos productos que España exporta con un nivel de especialización bajo o sin ventaja comparativa. Las lineas de división vertical y horizontal representan la densidad media y la ventaja comparativa revelada media de los productos, lo que permite dividir la figura en cuatro cuadrantes distintos. Todos aquellos productos situados en los cuadrantes segundo (C.II) y cuarto (C.IV) presentan un nivel de densidad por encima de la media, lo que implica un grado alto de cercanía con la estructura productiva existente y, en consecuencia, una mayor posibilidad de diversificación. Asimismo, los productos que se sitúan en el segundo cuadrante tienen un índice de ventaja comparativa revelada por encima de la media, y en muchos casos cercano a la unidad, lo que incrementa notablemente las posibilidades de diversificación. Por su parte, los productos situados en el tercer cuadrante (C.III) presentan un indice de ventaja comparativa y una densidad por debajo de la media, siendo así productos con bajas posibilidades de diversificación.

Figura 4

Densidad y ventaja comparativa revelada en productos de complejidad alta en España, $2014(\mathrm{VCR}<1)$

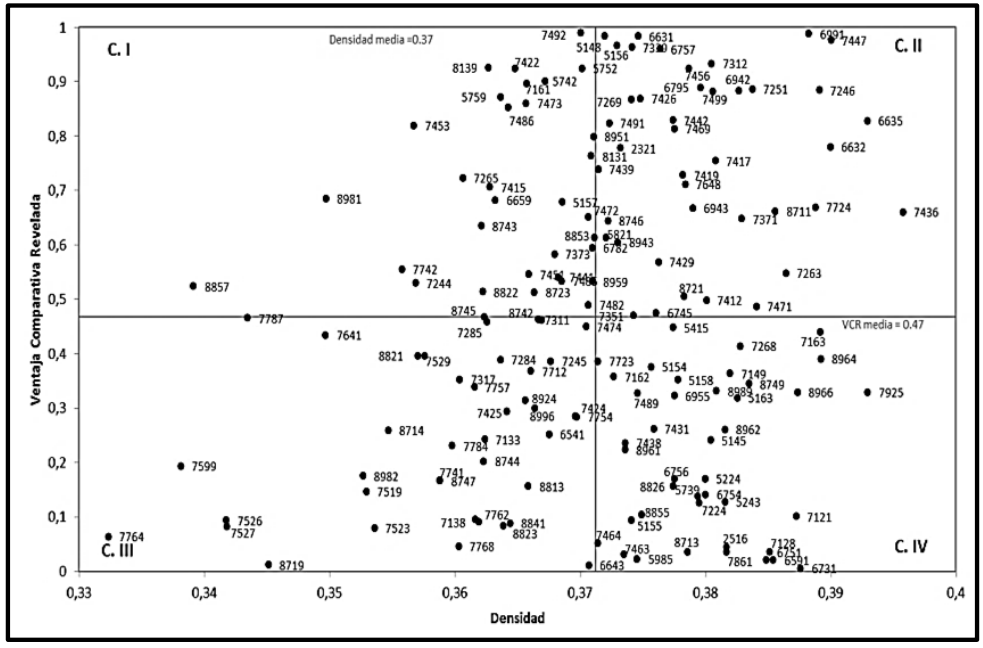

Fuente: Elaboración propia a partir de la base de datos UN COMTRADE.

Atendiendo exclusivamente a los productos con una densidad por encima de la media, se observa que predominan los productos pertenecientes a la división 74 (CUCI a 2 dígitos) de maquinaria y equipo industrial en general, partes y piezas de máquinas. Pues en efecto, el 28,8\% de los productos con una densidad por encima de la media pertenecen a la misma. Dentro de este grupo de 
productos destacan las máquinas y aparatos para la filtración y depuración de líquidos o gases (cod. 7436, VCR=0,66), los elevadores y transportadores de acción continua, para mercancías o materiales (cod. 7447, VCR=0,98), las válvulas reductoras de presión (cod. 7471, VCR=0,49.), los gasógenos y generadores de gas, plantas de destilación rectificación, unidades de intercambio térmico y máquinas para la licuefacción de aire u otros gases (cod. 7417, $\mathrm{VCR}=0,75$ ), las partes y piezas de máquinas, sin conectores, aisladores, bobinas, contactos y otros dispositivos eléctrico (cod. $7499, \mathrm{VCR}=0,88$ ) y los quemadores para alimentación de hogares, de combustible líquido, combustible sólido pulverizado, o gas (cod. 7412, VCR=0,50). Asimismo predominan los productos de la división 67 de hierro y acero (10,6\%), de la división 51 de productos químicos orgánicos (10,6\%), de la división 89 de artículos manufacturados diversos $(9,1 \%)$ y de la división 72 de maquinarias especiales para determinadas industrias $(9,1 \%)$.

Figura 5

Densidad y ventaja comparativa revelada en productos de complejidad media-alta en España, 2014 (VCR < 1)

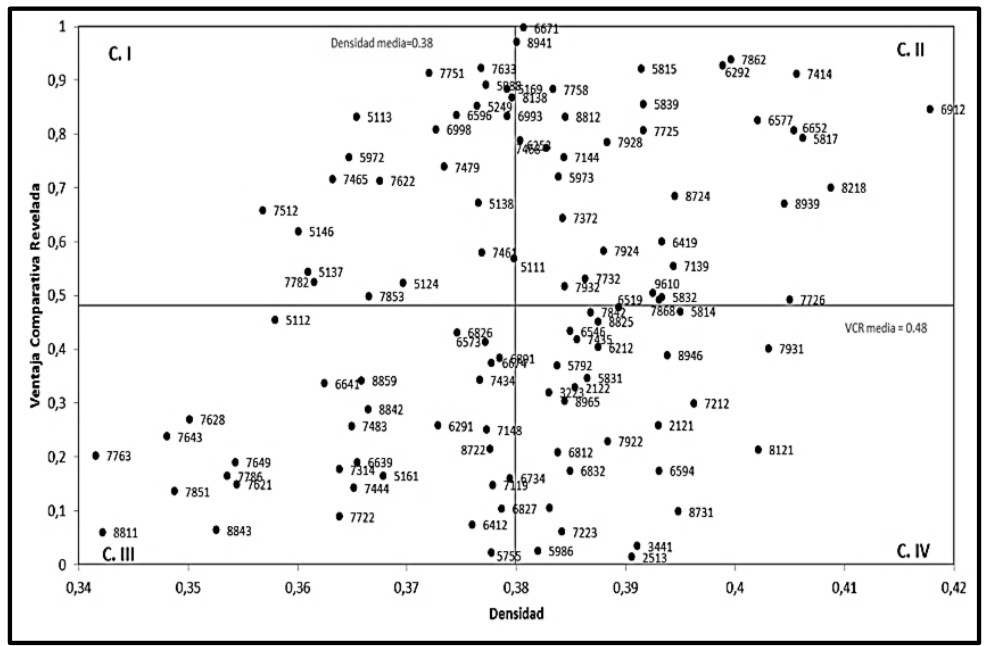

Fuente: Elaboración propia a partir de la base de datos UN COMTRADE.

En la Figura 5 se replica la Figura 4, para el conjunto de productos de complejidad media-alta. Atendiendo de nuevo exclusivamente a los productos con una densidad por encima de la media, el 10,2\% pertenecen a la división 58 de plásticos en formas no primarias, mientras que el 8,5\% se encuentran dentro de la división 79 de otro equipo de transporte. Las divisiones de artículos manufacturados diversos (cod. 89), maquinaria, aparatos y artefactos eléctricos, 
partes y piezas eléctricas (cod. 77) engloban, cada una de ellas, el 6,8\% de los productos.

En términos generales, tanto la Figura 4 como la Figura 5 ponen de manifiesto que los sectores que engloban una mayor proporción de productos en donde la posibilidad de diversificación es mayor son la maquinaria y equipo de transporte (sección 7, CUCI rev.3 a un dígito), los artículos manufacturados (sección 6 y sección 8) y los productos químicos y conexos (sección 5).

\section{IMPLICACIONES DE POLÍTICA ECONÓMICA}

El pensamiento sobre la política industrial ha seguido una senda zigzagueante en las últimas seis décadas. Desde la segunda guerra mundial hasta las crisis del petróleo, la tendencia dominante fue la intervención del sector público con un variado arsenal de políticas que incluían el apoyo a sectores concretos, los estímulos fiscales, las subvenciones, el proteccionismo por medio de barreras arancelarias y no arancelarias, y las compras públicas. La recuperación del pensamiento liberal y la oposición al keynesianismo condujeron a una redefinición de la política económica en general, y de la política industrial en particular. Una primera consecuencia fue el abandono de las políticas verticales (sectoriales). Se argumentaron razones de ineficacia, pues no quedaba demostrado que los sectores favorecidos resultaran económicamente más eficientes; se adujo además una dificultad casi insalvable para "elegir ganadores", pues tampoco quedaba claro cuáles deberían ser los criterios que el sector público tendría que aplicar para preferir ayudar a unas industrias en vez de a otras; y por último, se adujo también que las políticas verticales eran por su propia naturaleza, discriminatorias y distorsionadoras de la competencia. En consecuencia, las preferencias de la política pública se desplazaron hacia las llamadas políticas horizontales (transversales), que tenían al menos dos ventajas: una primera, según los análisis empíricos, apuntaba a que eran políticas que favorecían la eficiencia de la economía en su conjunto: por ejemplo, la política de dotación de infraestructuras de comunicaciones y transportes, redes informáticas etc.; y la política de fomento de la I+D+i y el desarrollo tecnológico. Una segunda ventaja era que, al no ser discriminatorias (por estar potencialmente al alcance de todas las empresas), no atentaban contra la competencia y no distorsionaban los precios relativos,

Pues bien, el trabajo que se presenta aquí tiene ciertas derivaciones aplicadas que sirven para superar algunas de las debilidades que se suelen achacar a las políticas verticales. Así en la línea de lo que corroboran algunos otros estudios ${ }^{11}$, se comprueba que las capacidades previas son significativamente importantes para aumentar la probabilidad de la diversificación industrial hacia nuevos

${ }^{11}$ Véase Donoso y Martín (2016). 
productos; es decir a productos que o no existían o carecían de VCR en el comercio exterior.

Aunque los mecanismos por los que opera la proximidad en cuanto palanca de la expansión productiva necesitan una mayor investigación, pueden apuntarse algunos factores que influyen: mencionemos la movilidad de los trabajadores entre industrias y territorios; la existencia de redes sociales de trabajo; los efectos de desbordamiento de las capacidades empresariales; la diversificación de las empresas y la proporción de gastos en $\mathrm{I}+\mathrm{D}+\mathrm{i}$ en relación con el producto nacional o regional.

Por tanto, aquellas políticas que contribuyan al fomento de estos factores tendrán un efecto positivo sobre la probabilidad de generar nuevos productos con VCR.

Al mismo tiempo, el análisis realizado nos proporciona un criterio fundado teórica y empíricamente, para "elegir ganadores" por los que pueden apostar las políticas públicas.

En concreto, del estudio empírico (ciñéndonos únicamente a los productos de mayor complejidad tecnológica) se desprenden indicaciones bastante precisas (aquí hemos llegado hasta los cuatro dígitos de la CUCI rev.3) acerca de qué productos ofrecería a la política industrial una mayor probabilidad de expandirse con VCR en el comercio exterior. Se ha apuntado a la sección 7 de maquinaria y equipo de transporte; y además las secciones 6 y 8, de artículos manufacturados, así como la sección 5 de productos químicos y conexos.

De lo anterior cabe concluir, según se ha apuntado en párrafos anteriores, que la metodología presentada permite tomar decisiones fundadas teórica y empíricamente, acerca de que productos o grupo de productos pueden ser apoyados con más alta probabilidad de desarrollar o ampliar su VCR en el comercio internacional.

\section{CONCLUSIONES}

En este trabajo, se ha analizado la complejidad económica de las exportaciones españolas, así como las posibilidades de diversificación hacia nuevos productos que permitan una mejora del nivel de complejidad de la economía. Los resultados obtenidos del análisis permiten obtener algunas conclusiones de interés.

En primer lugar, y a pesar del importante incremento en el número de productos que España exporta con ventaja comparativa que ha tenido lugar durante las últimas décadas, España presenta un nivel de complejidad relativamente bajo en comparación con los países de nuestro entorno más cercano. Además, el peso que representan las exportaciones de complejidad alta 
sobre el total de exportaciones, se ha reducido de forma considerable alcanzando valores muy inferiores a los observados en los países de nuestro entorno.

En segundo lugar se han identificado aquellos sectores en donde las posibilidades de diversificación hacia productos con un nivel de complejidad mayor son más elevadas. Tal es el caso de la maquinaria y equipo de transporte, los artículos manufacturados y productos químicos y conexos.

\section{REFERENCIAS BIBLIOGRÁFICAS}

ALONSO, J.A. y DONOSO, V. (1994). Competitividad de la empresa exportadora española. Madrid: Instituto Español de Comercio Exterior (ICEX).

ALONSO, J.A. y DONOSO, V. (2014). "El sector exterior en el proceso de modernización de la economía española: luces y sombras". En José Antono Alonso (coord.) y otros: Ensayos sobre economía española. Homenaje a José Luis García Delgado. Editorial Civitas (España), pp.185-200.

BALASSA, B. (1965). "Trade Liberalisation and Revealed Comparative Advantage". The Manchester School, 33, 99-123.

BOSCHMA, R., MINONDO, A. and NAVARRO, M. (2012). "The emergence of new industries at the regional level in Spain: a proximity approach based on product relatedness". Economic Geography, 89(1), pp. 29-51.

BRYCE, D. J., y WINTER, S. G. (2009). "A general interindustry relatedness index". Management Science, 55, pp. 1570-85.

CONSEJO ECONÓMICO Y SOCIAL (2012). "La internacionalización de la empresa española como factor de competitividad". Informe 02/2012, Madrid.

CORREA-LÓPEZ, M. y DOMÉNECH, R. (2012). "La internacionalización de las empresas españolas". Documentos de Trabajo, 12/29, BBVA, diciembre.

DONOSO, V. y MARTíN, V. (2014). "Evolución agregada y nivel tecnológico de las exportaciones españolas". Información Comercial Española, núm. 877, marzo-abril, pp. 9-25.

DONOSO, V. y MARTÍN, V. (2016). "Product relatedness and economic diversification in theUSA: an analysis at the state level". The Annals of Regional Science, Vol. 56 (2), marzo, pp. 449-471.

FAN, J. P. H. and LANG, L. H. P. (2000). "The measurement of relatedness: An application to corporate diversification". Journal of Business, 73, pp. 629-60.

FARJOUN, M. (1994). "Beyond industry boundaries: Human expertise, diversification and resource related industry groups". Organization Science, 5, pp.185-99.

GARCÍA TABUENCA, A. y CRECENTE, F. (2014). La internacionalización de la empresa española. Riesgos y oportunidades. Madrid: Fundación MAPFRE.

HAUSMANN, R. y KLINGER, B. (2007). "The structure of the product space and the evolution of comparative advantage". Working Paper $N^{\circ} 146$, Center for International Development at Harvard University. 
HIDALGO, C. (2009). "The dynamics of economic complexity and the product space over a 42 year period". Working Paper, No 189. Cambridge, MA: Center for International Development, Harvard University.

HIDALGO, C., y HAUSMANN, R. (2009). "The building blocks of economic complexity".

Proceedings of the National Academy of Sciences, 106(26), pp. 10570-10575.

HIDALGO, C.A., KLINGER, B., BARABÁSI, A.L. y HAUSMANN, R. (2007). "The product space conditions the development of nations". Science, 317, pp. 482-487.

MINISTERIO DE ECONOMÍA Y COMPETITIVIDAD (2016a). "El Sector Exterior en 2015". Boletin Económico de Información Comercial Española, núm. 3077.

MINISTERIO DE ECONOMÍA Y COMPETITIVIDAD (2016b). "Informe Mensual de Comercio Exterior". Agosto.

MINISTERIO DE ECONOMÍA Y COMPETITIVIDAD (2016c). "Análisis de la evolución reciente de los indicadores de competitividad-precio de la economía española(19902016)". Comercio Exterior, Estadísticas e Informes.

MINONDO, A. (2007). “¿Cuál es el grado de sofisticación de las exportaciones manufactureras españolas?". Estudios de Economía Aplicada, 25(3), pp. 643-654.

MYRO, Rafael (Dir.) y otros (2013). Fortalezas competitivas y sectores claves en la exportación española. Madrid: Instituto de Estudios Económicos.

NEFFKE F. M. H., y HENNING, M. (2008). "Revealed relatedness: Mapping industry space". Working Paper Series 08.19, Papers in Evolutionary Economic Geography, Utrecht, the Netherlands: Utrecht University.

NEFFKE, F., HENNING, M., y BOSCHMA, R. (2011). "How do regions diversify over time? Industry relatedness and the development of new growth paths in regions". Economic Geography, 87: 237-265.

TEECE, T. D.; RUMELT, R.; DOSI, G.; y WINTER, S. (1994). "Understanding corporate coherence: Theory and evidence". Journal of Economic Behaviour and Organization 23, pp. 1-30. 
